\title{
LOW ORDER COEFFICIENT ESTIMATES IN THE CLASS $\Sigma$
}

\author{
Y. J. LEUNG and G. SCHOBER
}

\section{Introduction}

In this article we study the coefficient functionals $b_{2}+\lambda b_{1}$ and $b_{3}+\lambda b_{1}$ in the class $\Sigma$ of univalent, analytic functions $f$ in $\tilde{\mathbf{D}}=\{z:|z|>1\}$ with the normalization

$$
f(z)=z+b_{0}+\frac{b_{1}}{z}+\frac{b_{2}}{z^{2}}+\ldots
$$

In general, $\lambda$ will be a complex constant.

We divide the paper into four sections. In Sections 1 through 3, we apply results from Jenkins' general coefficient theorem to describe the extremal functions for the linear functionals $b_{2}+\lambda b_{1}$ and $b_{3}+\lambda b_{1}$, respectively. By analyzing the associated Schiffer differential equations, the extremal values of the functionals are expressed at least implicitly. Finally, in Section 4 we discuss a coefficient conjecture for $\Sigma$ that originates from a paper by Chang, Schiffer, and Schober [2]. It is related to the nature of singularities lying on the trajectories of quadratic differentials associated with linear extremal problems.

\section{The functional $b_{2}+\lambda b_{1}$}

Let $L$ be a continuous linear functional defined on the space of analytic functions in $\tilde{\mathbf{D}}$ with the topology of locally uniform convergence. $L$ is assumed to be nonconstant on $\Sigma$ and $L(1)=0$. We say $f$ is a support point of $\Sigma$ for $L$ if $\operatorname{Re} L(f) \geqq$ $\operatorname{Re} L(g)$ for all $g$ in $\Sigma$. It is known (e.g., [10, Corollary 10.15]) that the omitted set of a support point $f$ consists of finitely many analytic arcs lying on trajectories of the quadratic differential $L(1 /(f-w)) d w^{2}$. The trajectories of this quadratic differential are analytic arcs on which $L(1 /(f-w)) d w^{2}>0$.

In this section we shall study the functional $L(f)=b_{2}+\lambda b_{1}$, where $\lambda$ is a fixed complex constant. In this case $L(1 /(f-w))=w-b_{0}+\lambda$ so that the associated quadratic differential is

$$
\left(w-b_{0}+\lambda\right) d w^{2} .
$$


We shall refer to

$$
b_{0}=\frac{1}{2 \pi} \int_{0}^{2 \pi} f\left(e^{i \theta}\right) d \theta
$$

as the conformal center of the omitted set of $f$.

The following result of Jenkins [4, Corollary 9] will be useful.

Lemma 1.1. Let $f \in \Sigma$ have the expansion (0.1) and map $\tilde{\mathbf{D}}$ onto the complement of arcs lying on trajectories of the quadratic differential $\left(w-b_{0}+\lambda\right) d w^{2}$. Then $\operatorname{Re}\left\{c_{2}+\lambda c_{1}\right\} \leqq \operatorname{Re}\left\{b_{2}+\lambda b_{1}\right\}$ for all $g(z)=z+c_{0}+c_{1} / z+\ldots$ in $\Sigma$. Equality holds only if $g=f+k$ for some constant $k$.

Since it is irrelevant what the value $b_{0}$ is, we choose $b_{0}=\lambda$. The resulting quadratic differential is $w d w^{2}$. Its critical trajectories are three rays emanating from the origin $w=0$ at angles $0,2 \pi / 3$, and $4 \pi / 3$, respectively. The case where the omitted arcs lie on the critical trajectories is completely contained in the following theorem and corollary of Jenkins [4, Corollary 10] and Kirwan and Schober [6, Theorem 7 and its corollary].

Theorem 1.2. If $f \in \Sigma$ has expansion (0.1) and $\lambda=(2 / 3)(a+b+c)$, where $|a|=$ $|b|=|c|=1$ and $a b c=1$, then

$$
\operatorname{Re}\left\{b_{2}+\lambda b_{1}\right\} \leqq \frac{2}{3}+\frac{1}{2}|\lambda|^{2}-\frac{1}{12} \operatorname{Re}\left\{\lambda^{3}\right\} .
$$

For each such $\lambda$ this inequality is sharp only for translations of

$$
f(z)=z[(1+a / z)(1+b / z)(1+c / z)]^{2 / 3} .
$$

The relation $\lambda=(2 / 3)(a+b+c)$ gives a one-to-one correspondence between the points $a, b, c$ of modulus one with $a b c=1$ and values $\lambda$ inside and on the hypocycloid with cusps at the points $2 e^{2 \pi k i / 3}, k=0,1,2$. The special case

$$
\operatorname{Re}\left\{b_{2}+2 e^{2 \pi k i / 3} b_{1}\right\} \leqq 2
$$

extends in an elementary way to the following.

Corollary 1.3. If $f \in \Sigma, t \geqq 2$, and $k=0,1,2$, then

$$
\operatorname{Re}\left\{b_{2}+t e^{2 \pi k i / 3} b_{1}\right\} \leqq t .
$$

This inequality is sharp only for translations of $f(z)=z+e^{-2 \pi k i / 3} z^{-1}$.

The purpose of this section is to study the situation where the omitted arc lies on a noncritical trajectory. Lemma 1.1 shows that any arc of conformal radius 1 lying on a trajectory of $w d w^{2}$ is the omitted set of a support point $f$ in $\Sigma$ supporting $L(f)=b_{2}+\lambda b_{1}$, where $\lambda$ is the conformal center of the arc. 
Assume that $f$ maps $\tilde{\mathbf{D}}$ onto the complement of an arc lying on a noncritical trajectory of $w d w^{2}$. Parametrizing the arc by $w=f\left(e^{i \theta}\right)$, we obtain

$$
f\left(e^{i \theta}\right)\left[i e^{i \theta} f^{\prime}\left(e^{i \theta}\right)\right]^{2}>0 .
$$

The Schwarz reflection principle gives

$$
f(z)\left[z f^{\prime}(z)\right]^{2}=q(z)=\frac{\left(z-e^{i \alpha}\right)^{2}\left(z-e^{i \beta}\right)^{2}\left(z-r e^{i \gamma}\right)\left(z-\frac{1}{r} e^{i \gamma}\right)}{z^{3}},
$$

where $\alpha, \beta, \gamma$ are real numbers and $0<r \leqq 1$. In addition, $q(z) \leqq 0$ on $|z|=1$.

The function $f$ maps $(1 / r) e^{i \gamma}$ to the origin $w=0$, which is the zero of the quadratic differential $w d w^{2}$. Since the boundary slit lies on a noncritical trajectory, the zero $(1 / r) e^{i \gamma}$ must lie in $\tilde{\mathbf{D}}$. Hence we have $0<r<1$.

The substitution of expansion (0.1) into (1.2) gives the following:

$$
\begin{gathered}
1=e^{2 i(\alpha+\beta+\gamma)}, \\
-\lambda=2\left(e^{i \alpha}+e^{i \beta}\right)+\left(r+\frac{1}{r}\right) e^{i \gamma}, \\
-b_{1}=e^{2 i \alpha}+e^{2 i \beta}+e^{2 i \gamma}+4 e^{i(\alpha+\beta)}+2\left(r+\frac{1}{r}\right)\left(e^{i \alpha}+e^{i \beta}\right) e^{i \gamma}, \\
2 \lambda b_{1}+3 b_{2}=2\left(e^{i \alpha}+e^{i \beta}\right)\left(e^{i(\alpha+\beta)}+e^{2 i \gamma}\right) \\
+\left(r+\frac{1}{r}\right)\left(e^{2 i \alpha}+e^{2 i \beta}+4 e^{i(\alpha+\beta)}\right) e^{i \gamma} .
\end{gathered}
$$

Since

$$
q\left(e^{i \theta}\right)=\frac{-16}{r} e^{i(\alpha+\beta+\gamma)} \sin ^{2}\left(\frac{\theta-\alpha}{2}\right) \sin ^{2}\left(\frac{\theta-\beta}{2}\right)\left|1-r e^{i(\theta-\gamma)}\right|^{2} \leqq 0,
$$

we have

$$
e^{i(\alpha+\beta+\gamma)}=1,
$$

which is consistent with (1.3). Therefore, using (1.7) to eliminate $\gamma$ in (1.4) to (1.6), we arrive at

$$
\begin{gathered}
-\lambda=2\left(e^{i \alpha}+e^{i \beta}\right)+2 s e^{-i(\alpha+\beta)}, \\
-b_{1}=e^{2 i \alpha}+e^{2 i \beta}+e^{-2 i(\alpha+\beta)}+4 e^{i(\alpha+\beta)}+4 s\left[e^{-i \alpha}+e^{-i \beta}\right], \\
2 \lambda b_{1}+3 b_{2}=8 \cos \frac{\alpha-\beta}{2} \cos \frac{3(\alpha+\beta)}{2}+4 s[2+\cos (\alpha-\beta)],
\end{gathered}
$$

where

$$
s=\frac{1}{2}\left(r+\frac{1}{r}\right)
$$

satisfies $s>1$. 
Now it is easy to represent

$$
\begin{aligned}
b_{2}+\lambda b_{1} & =\frac{1}{3}\left(2 \lambda b_{1}+3 b_{2}\right)+\frac{1}{3} \lambda b_{1} \\
& =\frac{8}{3} \cos \frac{\alpha-\beta}{2} \cos \frac{3(\alpha+\beta)}{2}+\frac{4}{3} s[2+\cos (\alpha-\beta)] \\
+\frac{2}{3}\left[e^{i \alpha}+e^{i \beta}\right. & \left.+s e^{-i(\alpha+\beta)}\right]\left[e^{2 i \alpha}+e^{2 i \beta}+e^{-2 i(\alpha+\beta)}+4 e^{i(\alpha+\beta)}+4 s e^{-i \alpha}+4 s e^{-i \beta}\right],
\end{aligned}
$$

and after some simplification the extreme value of the functional is

$$
\begin{aligned}
M=\operatorname{Re}\left\{b_{2}+\lambda b_{1}\right\}=\frac{4}{3}\left[7+4 s^{2}\right. & +2 \cos (\alpha-\beta)] \cos \frac{\alpha-\beta}{2} \cos \frac{3(\alpha+\beta)}{2} \\
& +\frac{2}{3} s[16+12 \cos (\alpha-\beta)+\cos 3(\alpha+\beta)] .
\end{aligned}
$$

At this stage the extreme value $M$ depends on three real parameters $\alpha, \beta$, and $s$. Equation (1.8) provides two real equations on them. Next a length condition will be obtained. This will determine $M$ implicitly.

Using $w=f(z)$, the differential equation (1.2) becomes

$$
w d w^{2}=q(z)\left(\frac{d z}{z}\right)^{2} .
$$

First, the left side generates the mapping

$$
\zeta=\int_{0}^{w} w^{1 / 2} d w=\frac{2}{3} w^{3 / 2}
$$

This mapping carries sectors $\Omega_{w}^{1}$ and $\Omega_{w}^{2}$ in the $w$-plane onto the upper and lower half-planes, respectively, as shown in Figure 1.

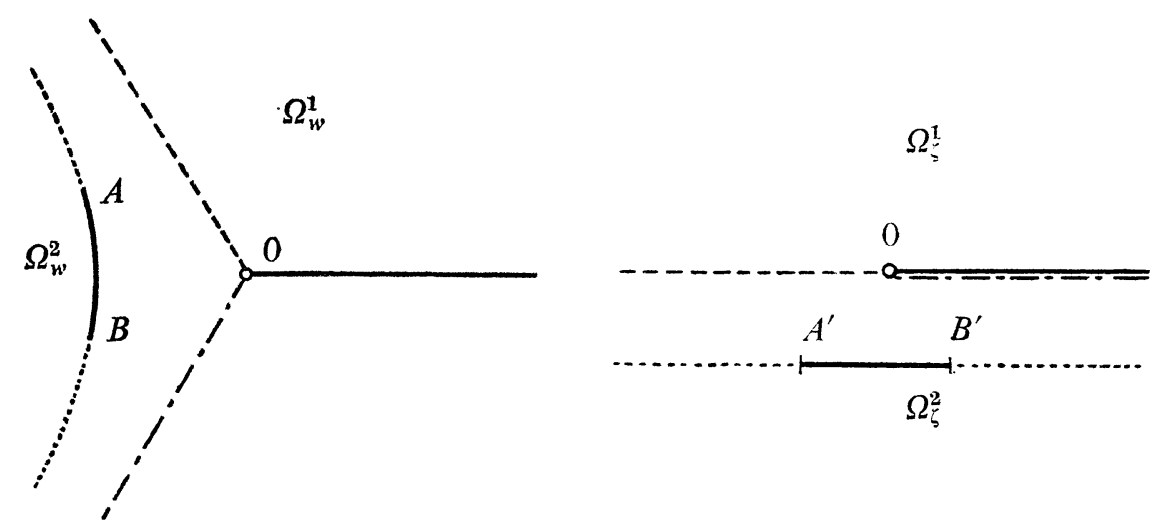

Figure 1 
There is a branch cut along the positive real axis in the $\zeta$-plane. An arc lying on a trajectory of $w d w^{2}$ in $\Omega_{w}^{2}$, for example, is mapped to a horizontal line segment in $\Omega_{\zeta}^{2}$.

For $q(z)(d z / z)^{2}$, there are double zeros at $e^{i \alpha}$ and $e^{i \beta}$ on $|z|=1$ and a simple one at $(1 / r) e^{i \gamma}$ in $\tilde{\mathbf{D}}$. The critical trajectories of $q(z)(d z / z)^{2}$ in $|z| \geqq 1$ are simulated in Figure 2.

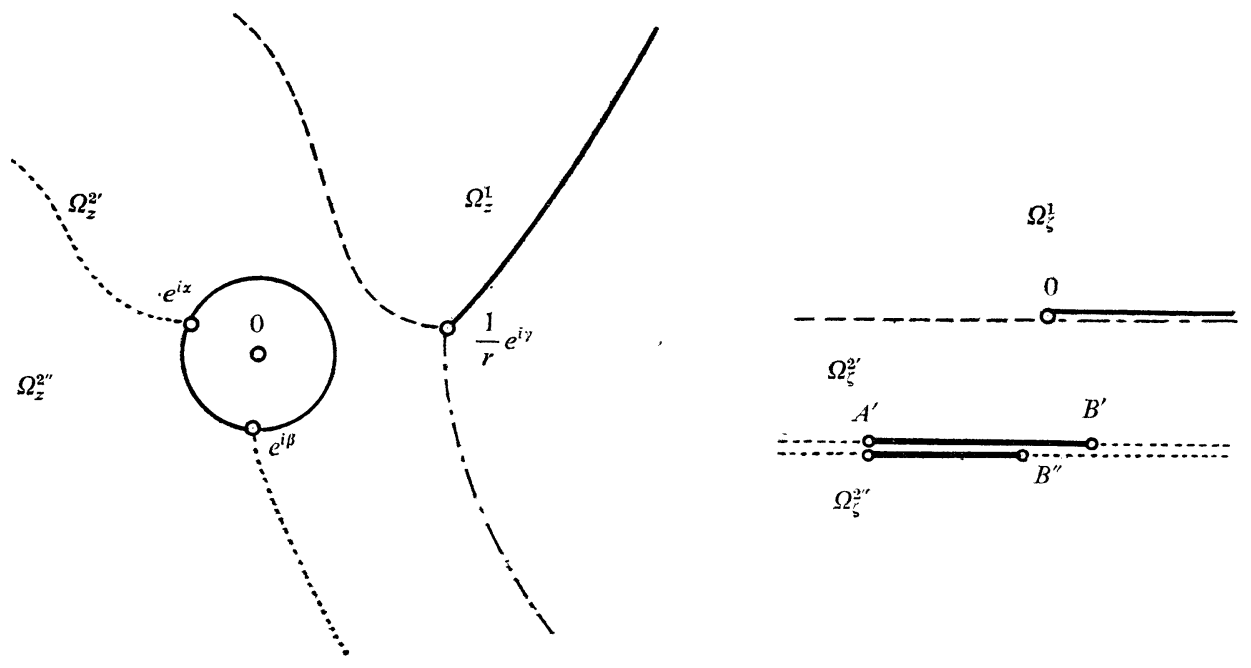

Figure 2

The mapping

$$
\zeta=\int_{(1 / r) e^{i_{\gamma}}}^{z} \sqrt{q(z)} \frac{d z}{z}
$$

transforms $\Omega_{z}^{1}$ onto the upper half $\zeta$-plane. The region $\Omega_{z}^{2}$ is mapped to a strip just below the real axis. The positive real axis is again a branch cut. The arc on the unit circle bordering $\Omega_{z}^{2^{\prime}}$ is mapped to a horizontal line segment $A^{\prime} B^{\prime}$ on the lower edge of the strip. If we continue from $\Omega_{z}^{2^{\prime}}$ into $\Omega_{z}^{2^{\prime \prime}}$ around the point $e^{i \alpha}$, which corresponds to $A^{\prime}$, then $\Omega_{z}^{2 \prime}$ will be mapped onto the half-plane below the line $A^{\prime} B^{\prime}$. In addition, the arc of the circle bordering $\Omega_{z}^{2^{\prime \prime}}$ will be mapped to a horizontal line segment $A^{\prime} B^{\prime \prime}$.

We shall impose the restriction

$$
\int_{|z|=1} \sqrt{q(z)} \frac{d z}{z}=0 .
$$

This forces $B^{\prime}$ and $B^{\prime \prime}$ to coincide and continuations around the outside of the circle $|z|=1$ to agree. Thus we can sew $\Omega_{\zeta}^{2^{\prime}}$ and $\Omega_{\zeta}^{2 \prime \prime}$ to obtain a single half-plane slit along $A^{\prime} B^{\prime}$. 
The two figures combine to give a mapping $f$ in $\Sigma$ from $|z|>1$ onto the $w$-plane slit along an arc lying on a trajectory in $\Omega_{w}^{2}$. By choosing other branches of the square roots, the slit will occur in the other sections of the $w$-plane.

If we assume that $\alpha<\beta<\alpha+2 \pi$, then (1.10) becomes

$$
\int_{\alpha}^{\beta}\left|q\left(e^{i \theta}\right)\right|^{1 / 2} d \theta=\int_{\beta}^{\alpha+2 \pi}\left|q\left(e^{i \theta}\right)\right|^{1 / 2} d \theta .
$$

This may be interpreted as a length condition since it says that the Euclidean lengths of $A^{\prime} B^{\prime \prime}$ and $A^{\prime} B^{\prime}$ agree. Since

$$
\left|q\left(e^{i \theta}\right)\right|=32 \sin ^{2}\left(\frac{\theta-\alpha}{2}\right) \sin ^{2}\left(\frac{\theta-\beta}{2}\right)[s-\cos (\theta+\alpha+\beta)],
$$

the length condition (1.11) can be written as

$$
\begin{aligned}
& -\sqrt{32} \int_{\alpha}^{\beta} \sin \left(\frac{\theta-\alpha}{2}\right) \sin \left(\frac{\theta-\beta}{2}\right) \sqrt{s-\cos (\theta+\alpha+\beta)} d \theta \\
= & \sqrt{32} \int_{\beta}^{\alpha+2 \pi} \sin \left(\frac{\theta-\alpha}{2}\right) \sin \left(\frac{\theta-\beta}{2}\right) \sqrt{s-\cos (\theta+\alpha+\beta)} d \theta,
\end{aligned}
$$

or, more simply,

$$
\int_{\alpha}^{\alpha+2 \pi} \sin \left(\frac{\theta-\alpha}{2}\right) \sin \left(\frac{\theta-\beta}{2}\right) \sqrt{s-\cos (\theta+\alpha+\beta)} d \theta=0 .
$$

This equation provides the last constraint on the parameters $\alpha, \beta$, and $s$.

In principle, the problem

$$
\max _{\Sigma} \operatorname{Re}\left\{b_{2}+\lambda b_{1}\right\}
$$

is now completely solved. If $\lambda$ is fixed and not on the hypocycloid or rays covered by Theorem 1.2 and Corollary 1.3, then $\lambda$ may be parametrized by equation (1.8), where $\alpha, \beta$, and $s$ satisfy the length condition (1.12). While this parametrization is not immediately obvious from relations (1.8) and (1.12), it is a consequence of our development. On the one hand, the problem (1.13) has a solution (1.9) that satisfies (1.8) and (1.12). On the other hand, each choice of parameters $\alpha, \beta, s$ satisfying the length condition (1.12) generates a function $f \in \Sigma$ that is admissible for the quadratic differential (1.1) with $\lambda$ defined by (1.8). Therefore (1.9) is the solution to the problem (1.13) for this choice of parameters. Let us summarize these conclusions.

Theorem 1.4. Let $\alpha, \beta$, and s be real numbers satisfying $\alpha<\beta<\alpha+2 \pi, s>1$, and

$$
\int_{\alpha}^{\alpha+2 \pi} \sin \left(\frac{\theta-\alpha}{2}\right) \sin \left(\frac{\theta-\beta}{2}\right) \sqrt{s-\cos (\theta+\alpha+\bar{\beta})} d \theta=0 .
$$


If $\lambda=-2 e^{i \alpha}-2 e^{i \beta}-2 s e^{-i(\alpha+\beta)}$, then

$$
\begin{aligned}
\max _{\Sigma} \operatorname{Re}\left\{b_{2}+\lambda b_{1}\right\}=\frac{4}{3}[7 & \left.+4 s^{2}+2 \cos (\alpha-\beta)\right] \cos \frac{\alpha-\beta}{2} \cos \frac{3(\alpha+\beta)}{2} \\
+ & \frac{2}{3} s[16+12 \cos (\alpha-\beta)+\cos 3(\alpha+\beta)] .
\end{aligned}
$$

Furthermore, every complex $\lambda$ not covered in Theorem 1.2 or Corollary 1.3 can be obtained in this way.

The extremal functions for (1.14) have all been described up to translation by our construction. Their omitted arcs lie on noncritical trajectories of the quadratic differentials (1.1).

All real values of $\lambda \geqq-2 / 3$ are covered by Theorem 1.2 and Corollary 1.3. Let us consider now just the real values $\lambda<-2 / 3$. In this case $e^{i \beta}=e^{-i \alpha}$ and the omitted arc for our extremal function lies symmetrically with respect to the negative real axis. Otherwise, we could switch the roles of $e^{i \alpha}$ and $e^{i \beta}$ to obtain another extremal function, in contradiction to the uniqueness statement in Lemma 1.1.

When $e^{i \beta}=e^{-i \alpha}$, the conditions (1.8), (1.9), and (1.12) can be simplified in terms of $x=\cos \alpha$ to

$$
\begin{gathered}
\lambda=-2 s-4 x \\
M=\frac{2}{3}\left[5 s+10 x+8 s^{2} x+24 s x^{2}+8 x^{3}\right], \\
\int_{0}^{2 \pi}(x-\cos \theta) \sqrt{s-\cos \theta} d \theta=0 .
\end{gathered}
$$

The latter could be written in terms of standard elliptic integrals, but it seems more informative in this way. Theorem 1.4 reduces to the following in this case.

Corollary 1.5. Let $s>1, \quad x=\int_{0}^{2 \pi}(\cos \theta) \sqrt{s-\cos \theta} d \theta / \int_{0}^{2 \pi} \sqrt{s-\cos \theta} d \theta$, and $\lambda=-2 s-4 x$. Then

$$
\max \operatorname{Re}\left\{b_{2}+\lambda b_{1}\right\}=\frac{2}{3}\left[5 s+10 x+8 s^{2} x+24 s x^{2}+8 x^{3}\right] .
$$

Furthermore, each $\lambda \in(-\infty,-2 / 3)$ is obtained in this way.

If $s \rightarrow+\infty$, then $x \rightarrow 1$ and $\lambda \rightarrow-\infty$. On the other hand, if $s \rightarrow 1$, then after some integrations one finds that $x \rightarrow-1 / 3$ and so $\lambda \rightarrow-2 / 3$. From these observations one has the final statement in Corollary 1.5 directly. 


\section{The functional $b_{3}+\lambda b_{1}$ for $\lambda$ in the diamond region}

For the convenience of computation and without loss of generality, we restrict our consideration of the functional $L(f)=b_{3}+\lambda b_{1}$ to the subclass $\Sigma_{0}$ of those functions in $\Sigma$ with the constant term missing. Since $L(1 /(f-w))=w^{2}-b_{1}+\lambda$, the omitted arc of a support point for such a functional lies on trajectories of the quadratic differential

$$
\left[w^{2}-\left(b_{1}-\lambda\right)\right] d w^{2} .
$$

We shall need a few technical results before coming to the problem itself. The structure of the critical trajectories of the differential $\left(w^{2}-\mu\right) d w^{2}$ will be analyzed first. Next, we show the oddness of the extremal function based on the uniqueness statement in the extended form of Jenkins' general coefficient theorem [5]. A special case of the general coefficient theorem is then applied to show that for $\lambda$ lying in a symmetric "diamond" region, the omitted arcs of a support point contain the two zeros of the quadratic differential (2.1). In this case we are able to solve the extremal problem

$$
\max _{\Sigma_{0}} \operatorname{Re}\left\{b_{3}+\lambda b_{1}\right\}
$$

fairly explicitly.

The quadratic differential $\left(w^{2}-\mu\right) d w^{2}, \mu \neq 0$, has simple zeros at $\pm \sqrt{\mu}$. If $\mu$ is purely imaginary, the two zeros are connected by a finite straight line trajectory through the origin. This can be easily demonstrated by substituting $w=\sqrt{\mu} t,-1 \leqq$ $t \leqq 1$, into the quadratic differential. The following converse result has been implicitly shown in Schaeffer and Spencer's monograph [9, Chapter 13], Ahlfors' book [1, Chapter 8], and Pfluger's article [8]. Our proof is different from theirs.

Lemma 2.1. If the zeros of $\left(w^{2}-\mu\right) d w^{2}, \mu \neq 0$, are connected by a critical trajectory, then $\mu$ must be purely imaginary.

Proof. There is only one finite critical trajectory joining $\sqrt{\mu}$ and $-\sqrt{\mu}$. Otherwise, two such trajectories would enclose a simply connected domain free of singularities. This domain would be mapped by $\zeta=\int_{\sqrt{\mu}}^{w} \sqrt{w^{2}-\mu} d w$ onto a bounded domain with a real boundary.

A trajectory joining $\sqrt{\mu}$ and $-\sqrt{\mu}$ must be symmetric with respect to the origin. Otherwise, its negative would be a second trajectory joining $\sqrt{\mu}$ and $-\sqrt{\mu}$. Consequently, there is an odd function $f(z)=\beta z+\beta_{1} / z+\ldots, \beta>0$, mapping $\tilde{\mathbf{D}}$ onto its complement. The function $f$ satisfies

$$
\left[f(z)^{2}-\mu\right]\left[z f^{\prime}(z)\right]^{2}=q(z)=\frac{\beta^{4}\left(z^{2}-e^{2 i \alpha}\right)^{4}}{z^{4}}
$$


for some real $\alpha$, and $q(z) \leqq 0$ on $|z|=1$. On the one hand,

$$
6 \beta^{4} e^{4 i \alpha}=\frac{1}{2 \pi} \int_{0}^{2 \pi} q\left(e^{i \theta}\right) d \theta<0 .
$$

On the other hand, a comparison of the $z^{2}$ terms on both sides gives

$$
\mu=4 \beta^{2} e^{2 i \alpha},
$$

which is evidently a purely imaginary number.

Next we show that the extremal functions for $L(f)=b_{3}+\lambda b_{1}$ are odd. For that purpose we shall use the following special case of Jenkins' general coefficient theorem [4, Theorem 3]. Very important for us will be the final uniqueness statement, which is taken from the extended form of the general coefficient theorem as found, for example, in [5]. Note that the quadratic differential (2.1) has a pole of order six at infinity.

Lemma 2.2 (Jenkins [4,5]). Let $\psi$ be univalent in a domain admissible for the quadratic differential $\left(w^{2}-\mu\right) d w^{2}$ and regular apart from a pole at infinity where it has the expansion

$$
\psi(w)=w+\frac{\alpha_{1}}{w}+\frac{\alpha_{2}}{w^{2}}+\frac{\alpha_{3}}{w^{3}}+\ldots
$$

Then

$$
\operatorname{Re}\left\{\alpha_{3}+(1 / 2) \alpha_{1}^{2}-\mu \alpha_{1}\right\} \leqq 0 .
$$

If equality holds in (2.3), then $\psi$ is a translation along the trajectories of $\left(w^{2}-\mu\right) d w^{2}$. If equality holds in (2.3) and $\alpha_{1}=0$, then $\psi(w) \equiv w$.

Lemma 2.3. If $f$ is an extremal function for the problem (2.2), then $f$ is odd.

Proof. Let $f(z)=z+b_{1} / z+b_{2} / z^{2}+\ldots$ be extremal for the problem (2.2) and define $g(z)=-f(-z)$. Then

$$
\psi(w)=g\left(f^{-1}(w)\right)=w-\frac{2 b_{2}}{w^{2}}-\frac{2 b_{4}+4 b_{1} b_{2}}{w^{4}}-\ldots
$$

is admissible for the quadratic differential $\left(w^{2}-\mu\right) d w^{2}$ with $\mu=b_{1}-\lambda$. Since equality holds in (2.3) and $\alpha_{1}=0$, we conclude from Lemma 2.2 that $\psi(w) \equiv w$. That is, $f$ is odd.

The oddness of $f$ can also be proved using the geometric ideas as presented in the Lemma of a paper by Charzyński and Schiffer [3] and in an article of Kubota [7]. See also Pfluger [8], who has considered a problem very similar to ours.

The following lemma contains additional a priori information.

Lemma 2.4. If $f(z)=z+b_{1} / z+\ldots$ is extremal for the problem (2.2), then $\left(\operatorname{Re} b_{1}\right)(\operatorname{Re} \lambda) \geqq 0$ and $\left(\operatorname{Im} b_{1}\right)(\operatorname{Im} \lambda) \leqq 0$. 
Proof. Compare $\operatorname{Re}\left\{b_{3}+\lambda b_{1}\right\}$ with $\operatorname{Re}\left\{\bar{b}_{3}-\lambda \bar{b}_{1}\right\}$ and $\operatorname{Re}\left\{\bar{b}_{3}+\lambda \bar{b}_{1}\right\}$, which arise from $i \overline{f(i \bar{z})}$ and $\overline{f(\bar{z})}$.

The omitted arcs of extremal functions for our present problem have two possible configurations. The first configuration contains a straight line segment connecting the two zeros of $\left(w^{2}-\mu\right) d w^{2}$. The second is a single arc symmetric with respect to the origin. They are indicated in Figure 3 for $\operatorname{Im} \mu<0$.

Align the pictures so that the 0 's are of the same leight.

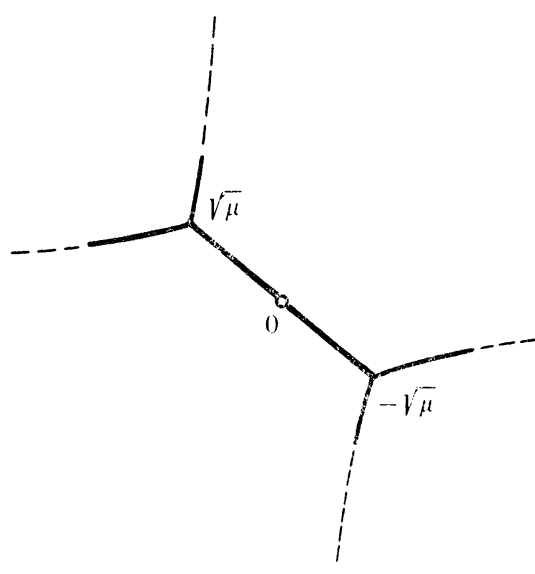

$\mu$ purely imaginary

Figure 3

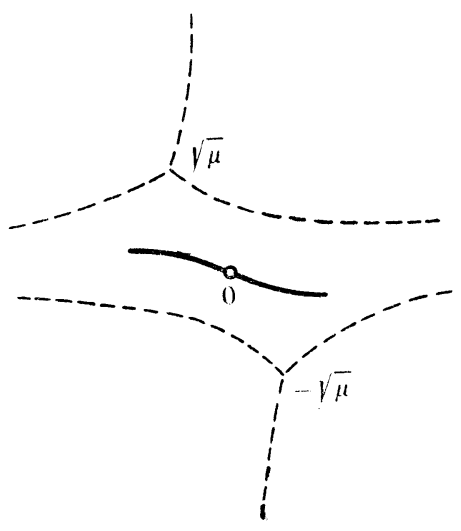

$\mu$ not purely imaginary

Next we develop the values for $\lambda$ that produce the first configuration. We shall see that these values of $\lambda$ lie in a diamond-shaped region. We shall make use of a method of Jenkins [4].

Lemma 2.5 (Jenkins [4, Lemma 4]). Let $Q(w) d w^{2}$ be the quadratic differential $\left(w^{2}+4 i s\right) d w^{2}$ with $0 \leqq s \leqq 1$. Then there is a family of odd functions $G(z, s, t) \in \Sigma_{0}$ which are admissible for $Q(w) d w^{2}$ and map $\tilde{\mathbf{D}}$ onto the complement of the straight line segment joining $2 \sqrt{s} e^{3 \pi i / 4}$ and $-2 \sqrt{s} e^{3 \pi i / 4}$ together with arcs of the other trajectories from these points. The real parameter $t$ measures the amount of translation along these other trajectories and satisfies the inequality

$$
|t| \leqq \sqrt{1-s^{2}}-s \arccos s .
$$

For $s=1$ only the straight line segment occurs. For $s=0$ the line segment degenerates to the origin and $G(z, 0, t)=z\left(1+2 t z^{-2}+z^{-4}\right)^{1 / 2}$. In any case the initial coefficients of $G(z, s, t)=z+\sum_{n=1}^{\infty} c_{n} z^{-n}$ are

$$
\begin{gathered}
c_{1}=t-i s(1-\log s), \\
c_{2}=0,
\end{gathered}
$$


and

$$
c_{3}=\frac{1}{2}\left[1-s^{2}-t^{2}+2 s^{2} \log s+s^{2}(\log s)^{2}\right]-i s t(1+\log s)
$$

with the appropriate limits for $s=0$.

If $f \in \Sigma_{0}$ and $\psi=f \circ G^{-1}$, then one obtains the following inequalities by combining Lemmas 2.2 and 2.5 .

Lemma 2.6 (Jenkins [4, Corollary 13]). If $f(z)=z+b_{1} / z+\ldots$ belongs to $\Sigma_{0}$, then

$$
\operatorname{Re}\left\{b_{3}+\frac{1}{2} b_{1}^{2}+4 i s b_{1}\right\} \leqq \frac{1}{2}+3 s^{2}-2 s^{2} \log s .
$$

Equality holds if and only if $f(z)$ is one of the functions $G(z, s, t)$.

We now define the diamond region mentioned earlier. For $e^{-3} \leqq s \leqq 1$, define

$$
\lambda(s)=\sqrt{1-s^{2}}-s \arccos s+i s(3+\log s) .
$$

This parametric curve appears in the first quadrant of the complex $\lambda$-plane and intercepts the axes at $\lambda\left(e^{-3}\right)=\sqrt{1-e^{-6}}-e^{-3} \arccos \left(e^{-3}\right) \approx .92303$ and $\lambda(1)=3 i$. The diamond region is obtained by reflecting the area enclosed to the other three quadrants. See Figure 4.

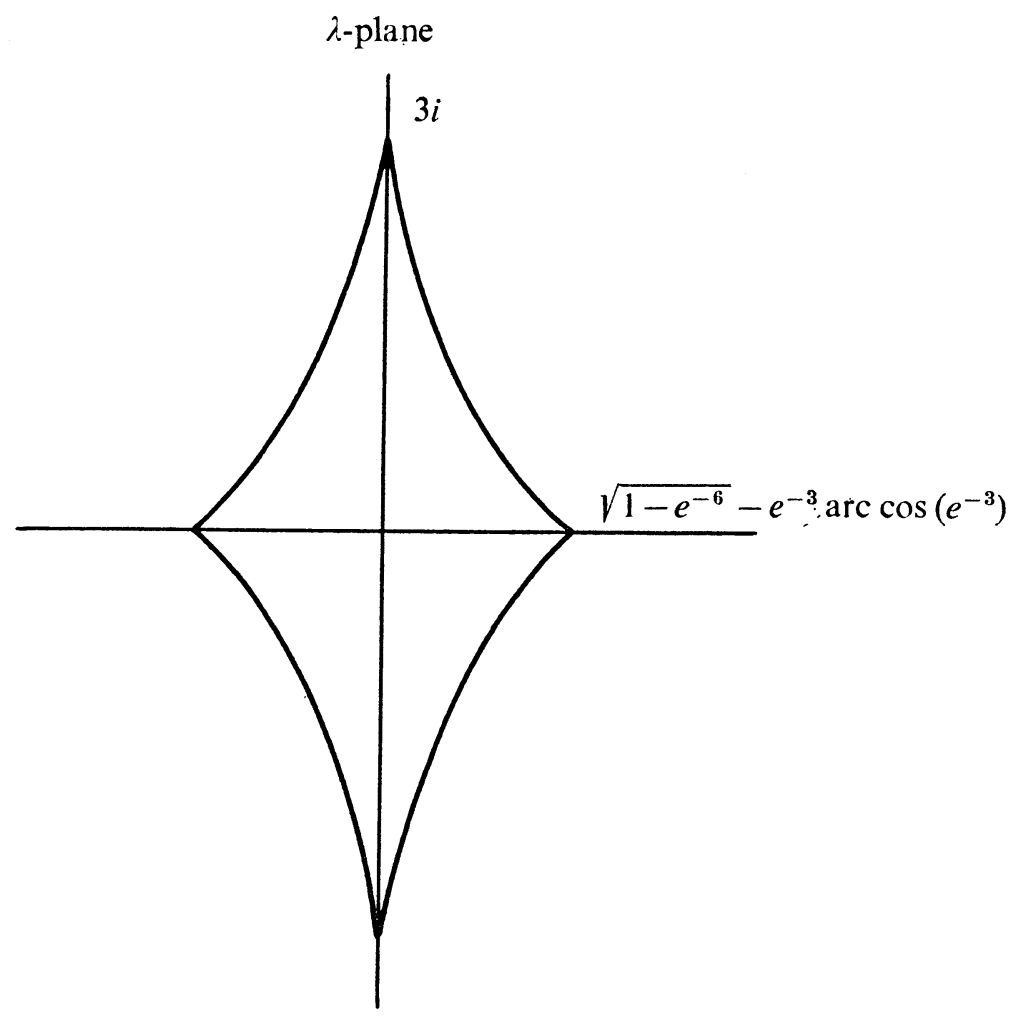

Figure 4 
Theorem 2.7. If $f(z)=z+\sum_{n=1}^{\infty} b_{n} z^{-n}$ belongs to $\Sigma_{0}$ and $\lambda$ is in the diamond region, then we have the sharp inequality

$$
\operatorname{Re}\left\{b_{3}+\lambda b_{1}\right\} \leqq \frac{1}{2}+\frac{1}{2}(\operatorname{Re} \lambda)^{2}-\frac{1}{2}(\operatorname{Im} \lambda)^{2}+\sigma^{2}+2 \sigma|\operatorname{Im} \lambda|
$$

where $\sigma$ is the unique solution of the equation

$$
s(3+\log s)=|\operatorname{Im} \lambda|
$$

in the interval $\left[e^{-3}, 1\right]$.

If, in addition, $\lambda$ is real, then

$$
\operatorname{Re}\left\{b_{3}+\lambda b_{1}\right\} \leqq \frac{1}{2}+e^{-6}+\frac{1}{2} \lambda^{2}
$$

and equality holds only when $f(z)$ is $G\left(z, e^{-3}, \lambda\right)$ or its conjugate $\overline{G\left(\bar{z}, e^{-3}, \lambda\right)}$. If $\lambda$ lies in the upper half-diamond, then equality holds in (2.4) only for $f(z)=G(z, \sigma, \operatorname{Re} \lambda)$. If $\lambda$ lies in the lower half-diamond, then equality holds only for $f(z)=\overline{G(\bar{z}, \sigma, \operatorname{Re} \lambda)}$.

Proof. By Lemma 2.6 we have

$$
\begin{aligned}
\operatorname{Re}\left\{b_{3}+\lambda b_{1}\right\} & =\operatorname{Re}\left\{b_{3}+\frac{1}{2} b_{1}^{2}+4 i s b_{1}\right\}-\operatorname{Re}\left\{\frac{1}{2} b_{1}^{2}+(4 i s-\lambda) b_{1}\right\} \\
& \leqq \frac{1}{2}+3 s^{2}-2 s^{2} \log s+\frac{1}{2}\left(\operatorname{Im} b_{1}\right)^{2}-\frac{1}{2}\left(\operatorname{Re} b_{1}\right)^{2} \\
& +(\operatorname{Re} \lambda)\left(\operatorname{Re} b_{1}\right)+(4 s-\operatorname{Im} \lambda) \operatorname{Im} b_{1}
\end{aligned}
$$

for $0 \leqq s \leqq 1$. Applying the inequality

$$
-\frac{1}{2} x^{2}+(\operatorname{Re} \lambda) x \leqq \frac{1}{2}(\operatorname{Re} \lambda)^{2}
$$

to $-(1 / 2)\left(\operatorname{Re} b_{1}\right)^{2}+(\operatorname{Re} \lambda)\left(\operatorname{Re} b_{1}\right)$, we obtain

$$
\operatorname{Re}\left\{b_{3}+\lambda b_{1}\right\} \leqq \frac{1}{2}+3 s^{2}-2 s^{2} \log s+\frac{1}{2}\left(\operatorname{Im} b_{1}\right)^{2}+\frac{1}{2}(\operatorname{Re} \lambda)^{2}+(4 s-\operatorname{Im} \lambda) \operatorname{Im} b_{1} .
$$

Assume now that $\operatorname{Im} \lambda \geqq 0$. If $\operatorname{Im} \lambda>0$, then it is sufficient to consider only the case $\operatorname{Im} b_{1} \leqq 0$ by Lemma 2.4. If $\operatorname{Im} \lambda=0$, then $\overline{f(\bar{z})}$ is also an extremal function whenever $f$ is, and so we may again assume $\operatorname{Im} b_{1} \leqq 0$. Since the function $-s(1-\log s)$ is strictly decreasing from 0 to -1 in the interval $[0,1]$, there is a unique $s$ such that

$$
\operatorname{Im} b_{1}=-s(1-\log s)=\operatorname{Im} c_{1} .
$$

With this choice for $s$ we have

$$
\operatorname{Re}\left\{b_{3}+\lambda b_{1}\right\} \leqq \frac{1}{2}-\frac{1}{2} s^{2}+s^{2} \log s+\frac{1}{2} s^{2}(\log s)^{2}+\frac{1}{2}(\operatorname{Re} \lambda)^{2}+s(1-\log s)(\operatorname{Im} \lambda) .
$$

Although we do not know $s$, we may bound our functional by $\max _{0 \leqq s \leqq 1} h(s)$ where $h(s)$ denotes the expression on the right hand side. In fact, this provides an upper bound whenever $\operatorname{Im} \lambda \geqq 0$.

Assume now that $\lambda$ is also in the diamond region. By analyzing $h^{\prime}(s)=$ $(\log s)(3 s+s \log s-\operatorname{Im} \lambda)$, we see that there is a unique solution $s=\sigma$ in $\left[e^{-3}, 1\right]$ 
of the equation

$$
s(3+\log s)=\operatorname{Im} \lambda
$$

as long as $0 \leqq \operatorname{Im} \lambda \leqq 3$ and that $h$ is increasing for $0 \leqq s \leqq \sigma$ and decreasing for $\sigma \leqq s \leqq 1$. Consequently,

$$
\operatorname{Re}\left\{b_{3}+\lambda b_{1}\right\} \leqq h(\sigma)
$$

where $\sigma \in\left[e^{-3}, 1\right]$ satisfies $\sigma(3+\log \sigma)=\operatorname{Im} \lambda$. Using the latter equation to eliminate the lc garithms in $h(\sigma)$, we cbtain the result (2.4). Furthermore, if $\operatorname{Im} \lambda=0$, then $\sigma=e^{-3}$ and (2.4) reduces to (2.6).

If $\lambda$ is in the diamond and $\operatorname{Im} \lambda \geqq 0$, then it is possible to choose $s=\sigma$ to satisfy (2.5) and to choose $x=\operatorname{Re} b_{1}$ to be $\operatorname{Re} \lambda$. In this case all inequalities become equalities so that the estimate (2.4) is sharp. In addition, equality occurs in Lemma 2.6 so that $f$ must be the function $G(z, \sigma, \operatorname{Re} \lambda)$. To remove the assumption that $\operatorname{Im} b_{1} \leqq 0$ when $\operatorname{Im} \lambda=0$, we must also allow the extremal function $\overline{G(\bar{z}, \sigma, \lambda)}$ when $\lambda$ is real.

Finally, if $\lambda$ is in the diamond and $\operatorname{Im} \lambda<0$, then all results follow from what has been proved by taking complex conjugates.

Geometrically, we have the following situation. Starting from $\lambda=0$, there are two extremal functions $G\left(z, e^{-3}, 0\right)$ and its conjugate $\bar{G}\left(z, e^{-3}, 0\right)$. Let us take $G\left(z, e^{-3}, 0\right)$, whose omitted set consists of a diagonal line segment joining $2 e^{-3 / 2} e^{3 \pi i / 4}$ and $-2 e^{-3 / 2} e^{3 \pi i / 4}$ plus two symmetric arcs protruding from these two points. We truncate the arcs symmetrically and dilate the remaining set to have conformal radius one. The length of the line segment becomes $4 \varrho e^{-3 / 2}, \varrho>1$. The new mapping function $G\left(z, \varrho^{2} e^{-3}, 0\right)$ becomes the support point for $L(f)=$ $b_{3}+\lambda b_{1}$ with $\lambda=\left[3 \varrho^{2} e^{-3}+\varrho^{2} e^{-3} \log \left(\varrho^{2} e^{-3}\right)\right] i$. If the two arcs are translated in opposite directions along the trajectories of $\left(w^{2}+4 \varrho^{2} e^{-3} i\right) d w^{2}$, then the mapping function is $G\left(z, \varrho^{2} e^{-3}, \operatorname{Re} \lambda\right)$ with

$$
|\operatorname{Re} \lambda| \leqq \sqrt{1-\varrho^{4} e^{-6}}-\varrho^{2} e^{-3} \arccos \left(\varrho^{2} e^{-3}\right) .
$$

It is the support point for $L(f)=b_{3}+\lambda b_{1}$ with $\operatorname{Im} \lambda=\left[3 \varrho^{2} e^{-3}+\varrho^{2} e^{-3} \log \left(\varrho^{2} e^{-3}\right)\right]$. Finally, when the length of the straight line segment is extended to four, the mapping function is $G(z, 1,0)=z-i / z$. It is the support point for $L(f)=b_{3}+3 i b_{1}$.

Corollary 2.8. If $f \in \Sigma_{0}$ and $t \geqq 3$, then $\operatorname{Re}\left\{b_{3} \pm i t b_{1}\right\} \leqq t$. Equality occurs only for $f(z)=z \mp i / z$.

Proof. $\operatorname{Re}\left\{b_{3}+i t b_{1}\right\}=\operatorname{Re}\left\{b_{3}+3 i b_{1}\right\}+(t-3) \operatorname{Re}\left\{i b_{1}\right\} \leqq 3+(t-3)=t . \quad$ Equality occurs only for $f(z)=z-i / z$. The other choice of signs can be obtained by conjugation.

The following theorem shows that we have exhausted the cases of the first configuration in Figure 3. 
Theorem 2.9. A support point for the functional $L(f)=b_{3}+\lambda b_{1}$ is admissible for some quadratic differential $\left(w^{2}-\mu\right) d w^{2}$ with $\mu$ purely imaginary (and nonzero) if and only if $\lambda$ lies in the diamond plus the imaginary axis.

Proof. In one direction, if $\lambda$ lies in the diamond, then Theorem 2.7 shows that any support point must be admissible for some quadratic differential $\left(w^{2} \pm 4 i \sigma\right) d w^{2}$ with $\sigma \in\left[e^{-3}, 1\right]$. If $\lambda$ lies on the imaginary axis, but not in the diamond, then Corollary 2.8 implies that the only support points are $f(z)=z \mp i / z$, which are admissible for $\left(w^{2} \pm 4 i\right) d w^{2}$, respectively.

Conversely, suppose that $f(z)=z+c_{1} / z+\ldots$ is a support point and is admissible for $\left(w^{2}+4 i s\right) d w^{2}$ with $0<s<1$. Then $f$ is odd by Lemma 2.3 and must be one of the functions $G(z, s, t)$ from Lemma 2.5. At the same time, Schiffer's differential equation asserts that $f=G$ must be admissible for the quadratic differential $\left[w^{2}-\left(c_{1}-\lambda\right)\right] d w^{2}$. Since the omitted set for $G$ splits at the zeros of $\left(w^{2}+4 i s\right) d w^{2}$, we conclude that $\left[w^{2}-\left(c_{1}-\lambda\right)\right] d w^{2}$ must have the same zeros, and so $4 i s=-c_{1}+\lambda$. Together with the condition $\left(\operatorname{Im} c_{1}\right)(\operatorname{Im} \lambda) \leqq 0$ from Lemma 2.4, this is just the parametrization for $\lambda$ in the diamond.

If $f$ is a support point, hence odd, and admissible for $\left(w^{2}+4 i s\right) d w^{2}$ with $s \geqq 1$, then $f(z)=z-i / z$. At the same time the Schiffer differential equation becomes $\left[w^{2}-(-i-\lambda)\right] d w^{2}>0$. At the origin, the quotient

$$
0<\frac{\left[w^{2}+i+\lambda\right] d w^{2}}{\left(w^{2}+4 i s\right) d w^{2}}=\frac{i+\lambda}{4 i s}
$$

shows that $\lambda$ lies on the imaginary axis.

The cases for $s<0$ can be proved similarly or by reflection.

In conclusion, when $\lambda$ lies in the diamond or on the imaginary axis, the corresponding support points are admissible for quadratic differentials $\left(w^{2}+4 i s\right) d w^{2}$, in fact, with $|s| \geqq e^{-3}$. When $\lambda$ lies outside the diamond and the imaginary axis, the corresponding support points are admissible for some quadratic differential $\left(w^{2}-\mu\right) d w^{2}$ with $\mu=b_{1}-\lambda$ lying off of the imaginary axis, except possibly $\mu=b_{1}-\lambda$ $=0$. However, the case $\mu=b_{1}-\lambda=0$ cannot occur because of Corollary 4.5. We remark that the proof of Corollary 4.5 depends on earlier sections only in the example constructed in the proof of Corollary 3.2.

\section{The functional $b_{3}+\lambda b_{1}$ for $\lambda$ outside the diamond region}

We now turn to the case where $\lambda$ lies outside the diamond region and imaginary axis. In this case we shall integrate the differential equation

$$
\left(w^{2}-\mu\right) d w^{2}>0
$$

and convert the extremal problem into a maximum problem with few parameters. 
A support point for the functional $L(f)=b_{3}+\lambda b_{1}$ must satisfy a differential equation of the form (3.1), in fact, with $\mu=b_{1}-\lambda$. For $\lambda$ outside the diamond and imaginary axis we have the important information at the conclusion of the previous section that it is sufficient to assume $\operatorname{Re} \mu \neq 0$. Thus we are in the situation of the second configuration in Figure 3.

If $f$ satisfies (3.1), then

$$
\left[f(z)^{2}-\mu\right]\left[z f^{\prime}(z)\right]^{2}=q(z)=\sum_{k=-4}^{4} q_{k} z^{k}
$$

where $q_{-k}=\bar{q}_{k}$ and $q(z) \leqq 0$ on $|z|=1$. For an odd function $f$, a comparison of the coefficients of $z^{k}$ on both sides shows that

$$
\begin{aligned}
& q_{4}=1, \\
& q_{3}=q_{1}=0, \\
& q_{2}=-\mu, \\
& q_{0}=-4 b_{3}-2 b_{1}^{2}+2 \mu b_{1} .
\end{aligned}
$$

Since $f^{\prime}$ vanishes at the two opposite points $\pm e^{i \alpha / 2}$ corresponding to the tips of the slit, $q$ can also be written as

$$
q(z)=\left(z^{2}-e^{i \alpha}\right)^{2}\left(1-\varrho e^{i \beta} z^{-2}\right)\left(1-\frac{1}{\varrho} e^{i \beta} z^{-2}\right)
$$

for some $\varrho \geqq 1$ and real $\beta$. In fact, $\varrho$ is strictly larger than one by Lemma 2.1 . We have $e^{2 i \alpha} e^{2 i \beta}=q_{-4}=1$, and so $e^{i \beta}= \pm e^{-i \alpha}$. The negative sign is the correct one since

$$
q\left(e^{i \beta / 2}\right)=\left(e^{i \beta}-e^{i \alpha}\right)^{2}(1-\varrho)\left(1-\frac{1}{\varrho}\right)<0 .
$$

So the final form of $q$ is

$$
q(z)=\left(z^{2}-e^{i \alpha}\right)^{2}\left(1+2 r e^{-i \alpha} z^{-2}+e^{-2 i \alpha} z^{-4}\right) \quad \text { with } \quad r=\frac{1}{2}\left(\varrho+\frac{1}{\varrho}\right)>1 .
$$

In addition,

and

$$
\mu=-q_{2}=2 e^{i \alpha}-2 r e^{-i \alpha}
$$

$$
2 b_{3}+b_{1}^{2}-\mu b_{1}=-\frac{1}{2} q_{0}=2 r-\cos 2 \alpha .
$$

Note that the correspondence between $\mu$ and $r e^{i \alpha}$ is one-to-one.

If we choose the zero $z=i \sqrt{\varrho} e^{-i \alpha / 2}$ to correspond to the zero $w=\sqrt{\mu}$, then integration of the differential equation

$$
\int_{\sqrt{\mu}}^{w} \sqrt{w^{2}-\mu} d w=\int_{i \sqrt{\varrho} e^{-i \alpha / 2}}^{z} \sqrt{q(z)} \frac{d z}{z}
$$


for $w=f(z)$ leads to the relation

$$
\begin{gathered}
w^{2} \sqrt{1-\mu w^{-2}}-\frac{\mu}{2} \log \left[\frac{w^{2}}{\mu}\left(2+2 \sqrt{1-\mu w^{-2}}-\mu w^{-2}\right)\right] \\
=\left(z^{2}+e^{i \alpha}\right) S-\frac{\mu}{2} \log \left[\frac{-e^{i \alpha} z^{2}}{\sqrt{r^{2}-1}}\left(S+1+r e^{-i \alpha} z^{-2}\right)\right] \\
-\frac{\bar{\mu}}{2} \log \left[\frac{S+r+e^{-i \alpha} z^{-2}}{\sqrt{r^{2}-1}}\right]
\end{gathered}
$$

where $S(z)=\left(1+2 r e^{-i \alpha} z^{-2}+e^{-2 i \alpha} z^{-4}\right)^{1 / 2}$. We substitute $f(z)=z+\sum_{n=1}^{\infty} b_{n} z^{-n}$ into the left side of the relation. The expansion around $\infty$ is

$$
\begin{gathered}
z^{2}+2 b_{1}-\frac{\mu}{2}-\frac{\mu}{2} \log \frac{4 z^{2}}{\mu}+\ldots \\
=z^{2}+e^{i \alpha}+r e^{-i \alpha}-\frac{\mu}{2} \log \frac{-2 e^{i \alpha} z^{2}}{\sqrt{r^{2}-1}}-\frac{\bar{\mu}}{4} \log \frac{r+1}{r-1}+\ldots,
\end{gathered}
$$

and a comparison of the constant terms gives

$$
b_{1}=e^{i \alpha}-\frac{\mu}{4} \log \frac{-\mu e^{i \alpha}}{2 \sqrt{r^{2}-1}}-\frac{\bar{\mu}}{8} \log \frac{r+1}{r-1} .
$$

It will be convenient to use polar coordinates for $-\mu / 2=\left(r e^{-i \alpha}-e^{i \alpha}\right)=R e^{i \gamma}$. Then $b_{1}=A+i B$ where

$$
\begin{gathered}
A=\cos \alpha+\frac{1}{2} R(\cos \gamma) \log \frac{R}{r-1}-\frac{1}{2} R(\alpha+\gamma) \sin \gamma \\
B=\sin \alpha+\frac{1}{2} R(\sin \gamma) \log \frac{R}{r+1}+\frac{1}{2} R(\alpha+\gamma) \cos \gamma \\
R=\sqrt{1+r^{2}-2 r \cos 2 \alpha} \\
\gamma=-\arctan \left(\frac{r+1}{r-1} \tan \alpha\right) .
\end{gathered}
$$

We shall use the formula $2 b_{3}=2 r-\cos 2 \alpha-b_{1}^{2}-2 R e^{i v} b_{1}$ to express

$$
b_{3}+\lambda b_{1}=r-\frac{1}{2} \cos 2 \alpha-\frac{1}{2} b_{1}^{2}+\left(\lambda-R e^{i \gamma}\right) b_{1}
$$

in terms of the parameters for $b_{1}$. That is,

$$
\operatorname{Re}\left\{b_{3}+\lambda b_{1}\right\}=r-\frac{1}{2} \cos 2 \alpha-\frac{1}{2} A^{2}+\frac{1}{2} B^{2}+(\operatorname{Re} \lambda-R \cos \gamma) A-(\operatorname{Im} \lambda-R \sin \gamma) B .
$$

Each complex $\mu$ with $\operatorname{Re} \mu \neq 0$ generates such a functional value, and the maximum is among them.

Since the maximum of our functional is the same for $\lambda$ and $\lambda$, it is no loss of generality to assume $\operatorname{Im} \lambda \geqq 0$. By Lemma 2.4 we may then also assume $\operatorname{Im} b_{1} \leqq 0$. 
It follows that we need only consider $\mu$ with $\operatorname{Im} \mu=\operatorname{Im}\left\{b_{1}-\lambda\right\} \leqq 0$. Therefore we shall restrict $0 \leqq \alpha \leqq \pi$ and $-\pi \leqq \gamma \leqq 0$.

Until now we did not specify the periods for $\alpha$ and $\gamma$ because we did not specify branches for the logarithms. That we have ultimately made the correct choices can be verified as in [9, pp. 223-225] by a continuity argument letting $r \rightarrow 1$.

One further restriction is possible. If we replace $\alpha$ by $\pi-\alpha$, then $\gamma$ is replaced by $-\pi-\gamma$, the expression $A$ changes sign, and $B$ is unchanged. As a result, in the expression for $\operatorname{Re}\left\{b_{3}+\lambda b_{1}\right\}$ only the term $(\operatorname{Re} \lambda) A$ changes sign. If we replace it by its absolute value, then we may restrict $0 \leqq \alpha \leqq \pi / 2$. Moreover, $\alpha$ cannot be $\pi / 2$ since we assumed $\operatorname{Re} \mu \neq 0$. Let us summarize and at the same time eliminate $R$ from the formulas.

Theorem 3.1. If $\lambda$ does not belong to the diamond region or imaginary axis, then

$$
\begin{gathered}
\max _{\Sigma_{0}} \operatorname{Re}\left\{b_{3}+\lambda b_{1}\right\}=\max _{\substack{0 \leq \alpha<\pi / 2 \\
r>1}}\left\{r-\frac{1}{2} \cos 2 \alpha-\frac{1}{2} A^{2}+\frac{1}{2} B^{2}+|A \operatorname{Re} \lambda|\right. \\
-B|\operatorname{Im} \lambda|-(r-1) A \cos \alpha-(r+1) B \sin \alpha\}
\end{gathered}
$$

where

$$
\begin{aligned}
& A=\cos \alpha+\frac{1}{2}(r-1)(\cos \alpha) \log \frac{\sqrt{1+r^{2}-2 r \cos 2 \alpha}}{r-1}+\frac{1}{2}(r+1)(\alpha+\gamma) \sin \alpha, \\
& B=\sin \alpha-\frac{1}{2}(r+1)(\sin \alpha) \log \frac{\sqrt{1+r^{2}-2 r \cos 2 \alpha}}{r+1}+\frac{1}{2}(r-1)(\alpha+\gamma) \cos \alpha
\end{aligned}
$$

and

$$
\gamma=-\arctan \left(\frac{r+1}{r-1} \tan \alpha\right) \in(-\pi / 2,0] .
$$

A particularly important choice for $\lambda$ is $\lambda=1$.

Corollary 3.2. $\operatorname{Max}_{\Sigma_{0}} \operatorname{Re}\left\{b_{3}+b_{1}\right\} \geqq 1.00124$.

Proof. Choose $\alpha=0.03508$ and $r=1.01710$ as competitors for the maximum in Theorem 3.1.

The numerical values in Corollary 3.2 were chosen by a search procedure. They are, presumably, very close to the optimal ones. We remark that $b_{1}$ cannot be real in this example since it was shown in [11] that

$$
\operatorname{Re}\left\{b_{3}+t b_{1}\right\} \leqq \frac{1}{2}\left(1+t^{2}\right)
$$

whenever $b_{1}$ is real and $-1 \leqq t \leqq 1$.

Corollary 3.2 shows that the Koebe function $k(z)=z+1 / z$ is not extremal for the problem $\max _{\Sigma_{0}} \operatorname{Re}\left\{b_{3}+b_{1}\right\}$. This fact will be important in Section 4. On the other hand, the following theorem shows that the Koebe function is extremal when $\lambda$ is real and somewhat larger than one. 
Theorem 3.3. There is a constant $\lambda_{0}, 1<\lambda_{0} \leqq 2$, such that

$$
\max _{\Sigma_{0}} \operatorname{Re}\left\{b_{3}+\lambda b_{1}\right\}=\lambda
$$

for all real $\lambda \geqq \lambda_{0}$. For $\lambda>\lambda_{0}$ the Koebe function $k(z)=z+1 / z$ is the only extremal function.

Proof. Consider first $\lambda=2$. By Lemma 2.3 an extremal function $f(z)=z+$ $b_{1} / z+\ldots$ for the problem (2.2) must be odd, and so

$$
f(\sqrt{z})^{2}=z+2 b_{1}+\left(2 b_{3}+b_{1}^{2}\right) / z+\ldots
$$

is a function in $\Sigma$. Therefore $\operatorname{Re}\left\{2 b_{3}+b_{1}^{2}\right\} \leqq 1$, and since $\operatorname{Re}\left\{b_{1}^{2}\right\} \geqq 2\left(\operatorname{Re} b_{1}\right)^{2}-1$, we have

$$
\operatorname{Re}\left\{b_{3}+2 b_{1}\right\}=\operatorname{Re}\left\{b_{3}+\frac{1}{2} b_{1}^{2}\right\}+2 \operatorname{Re} b_{1}-\frac{1}{2} \operatorname{Re}\left\{b_{1}^{2}\right\} \leqq \frac{1}{2}+\frac{3}{2}-\left(1-\operatorname{Re} b_{1}\right)^{2} \leqq 2 .
$$

Although we shall not use it, note that equality occurs only for $k(z)=z+1 / z$.

We have shown that (3.2) holds for $\lambda=2$. If (3.2) holds for some $\lambda_{0}$, then it holds for all $\lambda>\lambda_{0}$ since

$$
\operatorname{Re}\left\{b_{3}+\lambda b_{1}\right\}=\operatorname{Re}\left\{b_{3}+\lambda_{0} b_{1}\right\}+\left(\lambda-\lambda_{0}\right) \operatorname{Re} b_{1} \leqq \lambda_{0}+\left(\lambda-\lambda_{0}\right)=\lambda .
$$

Furthermore, equality for some $\lambda>\lambda_{0}$ can occur only if $\operatorname{Re} b_{1}=1$ or if the function is the Koebe function.

By compactness of $\Sigma_{0}$ and Corollary 3.2 there will be a least $\lambda_{0}$ for which (3.2) holds, and it must satisfy $1<\lambda_{0} \leqq 2$.

Theorem 3.3 asserts that the Koebe function is the unique extremal function for $\lambda>\lambda_{0}$. We conjecture that it is the unique extremal function for $\lambda=\lambda_{0}$, too. If this is true, then one can deduce from the representation for $b_{1}$ that $\lambda_{0}=$ $\left(e^{4}+3\right) /\left(e^{4}-1\right)=1.0746 \ldots$ This value corresponds to $\lambda=b_{1}-\mu$ for the parameter values $\alpha=0$ and $r=\left(e^{4}+1\right) /\left(e^{4}-1\right)$.

At this stage, we have shown for $\lambda$ in the open interval

$$
\left(\sqrt{1-e^{-6}}-e^{-3} \arccos \left(e^{-3}\right), \lambda_{0}\right)
$$

that the corresponding extremal functions map the unit circle onto part of a regular trajectory of $\left(w^{2}-\mu\right) d w^{2}$ with $\mu$ neither real nor purely imaginary (cf. the second configuration in Figure 3). If $\lambda_{0}=\left(e^{4}+3\right) /\left(e^{4}-1\right)$, then this interval is just $(.92303 \ldots, 1.0746 \ldots)$.

Another reasonable conjecture is that there are exactly two extremal functions $f(z)$ and $\overline{f(\bar{z})}$ for each $\lambda$ in this interval. If true, then it appears that the functional $L(f)=b_{3}+\lambda b_{1}$, for $\lambda$ in the first or fourth quadrant outside the diamond, can be obtained via truncation from a unique $\lambda$ in this interval. 


\section{A coefficient conjecture}

In this last section we shall consider general linear problems for $\Sigma$. The omitted sets for corresponding support points consist of finitely many analytic arcs. Points of nonanalyticity or forking can occur only at zeros of the associated quadratic differential $L(1 /(f-w)) d w^{2}$. Various examples, as in Sections 1 and 2, show that forking can occur. The purpose of this section is to conjecture that only one type of forking can occur, to reduce this conjecture to some coefficient conjectures, and to observe that we have already solved one of these conjectures in Section 3.

In contrast, in the familiar class $S$ of normalized univalent functions in the unit disk, the omitted set of a support point is a single analytic arc extending to $\infty$. The arc does not contain any of the critical points of the quadratic differential, except possibly a zero at its finite tip. In this exceptional case the arc is a radial line. This is due essentially to the fact that the omitted set can be transformed by Möbius transformations to omitted sets of other functions in $S$. In $\Sigma$, the only Möbius transformations allowed are trivial Euclidean translations.

Let $L$ be a continuous linear functional with the same assumptions stated at the beginning of Section 1 . The omitted set $\Gamma$ of a corresponding support point $f \in \Sigma$ satisfies $L(1 /(f-w)) d w^{2}>0$. The function $L(1 /(f-w))$ extends to a nonconstant analytic function of $w$ in a neighborhood of $\Gamma$ and hence has at most finitely many zeros on $\Gamma$. At a zero of order $k \geqq 1$ there can be at most $k+2$ arcs emanating under equal angles from that point. If $k \geqq 2$, it was shown in [2, pp. 216-218] that all $k+2$ arcs cannot occur. Furthermore, under additional hypotheses the angle between arcs must be at least $2 \pi / 5$.

Various examples, as in Sections 1 and 2 show that simple zeros can occur on $\Gamma$, but such zeros are the only ones known actually to occur. Therefore the following may be reasonable.

Conjecture 4.1. The quadratic differential $L(1 /(f-w)) d w^{2}$ corresponding to a support point $f$ of $\Sigma$ can have only simple zeros on the omitted set $\Gamma$.

If the conjecture is true, then $\Gamma$ can fork at each simple zero in at most three directions at equal angles. Note that forking actually occurs in only two of the three directions for $\lambda$ on the boundary of the hypocycloid in Section 1 and for $\lambda$ on the boundary of the diamond in Section 2.

In the following, we shall show that Conjecture 4.1 is equivalent to some coefficient conjectures. The method is similar to that of [2].

With no loss of generality, suppose that $w=0$ is a point lying in the omitted set $\Gamma$ of a support point $f$ in $\Sigma$ for $L$. Assume that $w=0$ is a zero of order $k$ for the function $L(1 /(f-w))$. This implies that $L\left(f^{-j}\right)=0$ for $j=0,1, \ldots, k$ and that $L\left(f^{-k-1}\right) \neq 0$. Without loss of generality we can assume $L\left(f^{-k-1}\right)>0$. This implies that the asymtotic directions of $\Gamma$ at the origin are a subset of the directions determined by the $(k+2)^{\text {th }}$ roots of unity. 
We start by deleting a few, or possibly none, of the arcs of $\Gamma$ coming out from $w=0$. Designate the further subcontinuum inside $|w| \leqq \delta$ and containing $w=0$ by $\Gamma_{\delta}$. Let $F_{\delta}$ map $\tilde{\mathbf{D}}$ onto the complement of $\Gamma_{\delta}$ with the expansion $F_{\delta}(z)=\varrho z+$ $O(1)$ at infinity. Then $F_{\delta}$ and the original $f$ are related by a univalent "Schwarz function" $\omega$ satisfying $F_{\delta} \circ \omega=f$ in $\tilde{\mathbf{D}}$. The function $\omega$ maps $\tilde{\mathbf{D}}$ onto $\tilde{\mathbf{D}}$ minus a few slits growing out from $|z|=1$. In addition, $\omega^{\prime}(\infty)=1 / \varrho>1$ and $\varrho \rightarrow 0$ as $\delta \rightarrow 0$.

The function $f_{\delta}=(1 / \varrho) F_{\delta}$ is a support point for the functional $L_{\delta}$ defined by $L_{\delta}(g)=L(\varrho g \circ \omega)$. The omitted set for $f_{\delta}$ is denoted by $\gamma_{\delta}$, and it results from dilating $\Gamma_{\delta}$ by the factor $1 / \varrho$. As $\delta \rightarrow 0, \gamma_{\delta}$ approaches a set $\gamma_{0}$ consisting of several radial line segments of equal length emanating from the origin.

Let $\psi$ be univalent in the complement of $\gamma_{\delta}$ and have an expansion $\psi(w)=$ $w+\sum_{j=0}^{\infty} c_{j}(\delta) w^{-j}$ in a neighborhood of infinity. Then $f_{\delta}^{*}=\psi \circ f_{\delta}$ serves as a variation of $f_{\delta}$ and so $\operatorname{Re} L_{\delta}\left(f_{\delta}^{*}\right) \leqq \operatorname{Re} L_{\delta}\left(f_{\delta}\right)$. In other words, $\operatorname{Re} L\left(f_{\delta}^{*} \circ \omega-f_{\delta} \circ \omega\right) \leqq 0$. Since

$$
f_{\delta}^{*} \circ \omega-f_{\delta} \circ \omega=\sum_{j=0}^{\infty} c_{j}(\delta) \varrho^{j} f^{-j}
$$

for $|f|>\delta$, it follows that $\operatorname{Re}\left\{\sum_{j=0}^{\infty} c_{j}(\delta) \varrho^{j} L\left(f^{-j}\right)\right\} \leqq 0$ if $\delta$ is sufficiently small. Since $L\left(f^{-j}\right)=0$ for $j=0, \ldots, k$, we have

$$
\operatorname{Re}\left\{c_{k+1}(\delta)\right\} \varrho^{k+1} L\left(f^{-k-1}\right)+O\left(\varrho^{k+2}\right) \leqq 0
$$

where $L\left(f^{-k-1}\right)$ is positive. Dividing by $\varrho^{k+1} L\left(f^{-k-1}\right)$ and letting $\delta \rightarrow 0$, we obtain $\operatorname{Re}\left\{c_{k+1}\right\} \leqq 0$ for an arbitrary univalent function $\psi(w)=w+\sum_{j=0}^{\infty} c_{j} w^{-j}$ in the complement of $\gamma_{0}$. Let us summarize:

If the omitted set $\Gamma$ of a support point $f$ for some functional L contains a $k^{\text {th }}$ order zero of its associated quadratic differential $L(1 /(f-w)) d w^{2}$ and if $L\left((f-w)^{-k-1}\right)>0$ there, then one concludes that

$$
\operatorname{Re} c_{k+1} \leqq 0
$$

for every function $\psi$ analytic and univalent in the complement of $\gamma_{0}$ with expansion $\psi(w)=w+\sum_{j=0}^{\infty} c_{j} w^{-j}$ in a neighborhood of infinity.
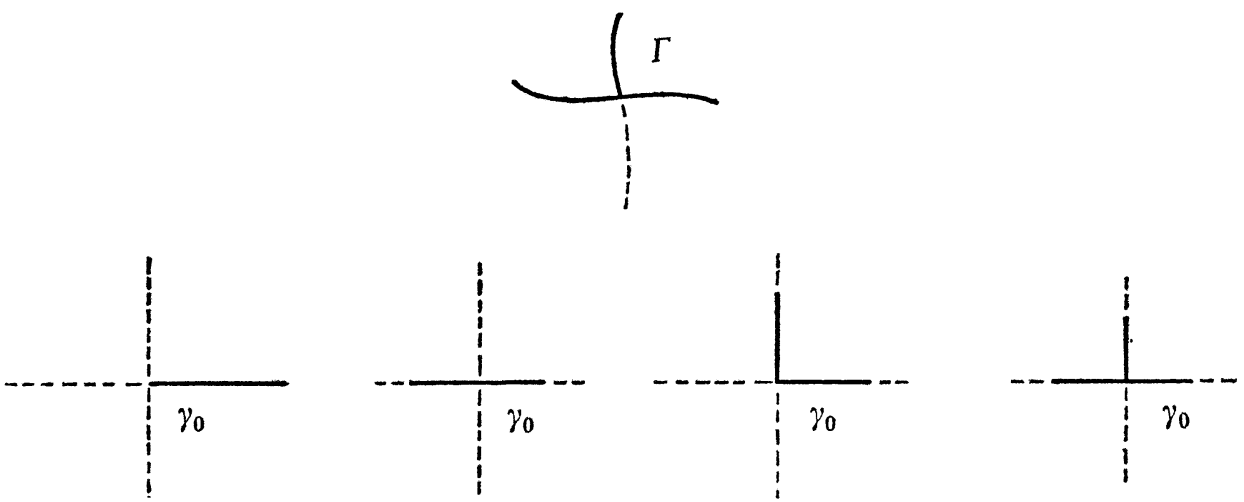

Figure 5 
Since $\Sigma$ admits translations and rotations, we may assume that $\gamma_{0}$ consists of equal radial line segments emanating from the origin in a subset of the directions determined by the $(k+2)^{\text {th }}$ roots of unity. For example, in Figure 5 the arc $\Gamma$ contains a zero of order 2 , and the four essentially different choices for $\gamma_{0}$ are displayed.

The setting of the coefficient inequality (4.1) is quite similar to a result of Teichmüller [12]. Each assumption on the order of the zero and the geometric configuration of $\gamma_{0}$ implies a coefficient estimate (4.1). However, if we believe Conjecture 4.1, then there is no reason why the coefficient estimates (4.1) should be true for $k \geqq 2$. In fact, to verify Conjecture 4.1 it is sufficient to show that the estimates (4.1) are false.

\section{Conjecture 4.2. The estimates (4.1) are not valid for any $k \geqq 2$.}

Thus Conjecture 4.2 implies Conjecture 4.1. In fact, it is sufficient to verify Conjecture 4.2 when $\gamma_{0}$ is the real interval [0,4]. Furthermore, for each integer $k$ and configuration $\gamma_{0}$ that (4.1) is proved false there is a corresponding zero and configuration that cannot happen for any support point of $\Sigma$.

Let us translate the statement (4.1) into a coefficient statement for the class $\Sigma$. Let $\varphi_{0}(z)=z+\sum_{n=0}^{\infty} a_{n} z^{-n}$ map $\tilde{\mathbf{D}}$ onto the complement of $\gamma_{0}$, and let $\Phi_{0}$ denote its inverse function. If $g(z)=z+\sum_{n=0}^{\infty} b_{n} z^{-n}$ is an arbitrary function of $\Sigma$, then

$$
\psi(w)=g \circ \Phi_{0}(w)=\Phi_{0}(w)+\sum_{n=0}^{\infty} b_{n} \Phi_{0}(w)^{-n}=w+\sum_{j=0}^{\infty} c_{j} w^{-j}
$$

near infinity, and the statement (4.1) asserts that $\operatorname{Re} c_{k+1} \leqq 0$. Evidently,

$$
\begin{gathered}
c_{k+1}=b_{k+1}+\pi_{-1}\left(a_{0}, \ldots, a_{k+1}\right)+\sum_{j=1}^{k} \pi_{j}\left(a_{0}, \ldots, a_{k-j}\right) b_{j} \\
=\frac{1}{2 \pi i} \int_{|w|=R} g\left(\Phi_{0}(w)\right) w^{k} d w
\end{gathered}
$$

for $R$ sufficiently large. Each $\pi_{j}$ is a polynomial of its arguments and equals the coefficient of $w^{-k-1}$ in the expansion of $\Phi_{0}^{-j}$ at infinity.

Thus (4.1) is equivalent to the linear coefficient inequality

$$
\operatorname{Re}\left\{b_{k+1}+\pi_{-1}+\sum_{j=1}^{k} \pi_{j} b_{j}\right\} \leqq 0
$$

for every $g(z)=z+\sum_{n=0}^{\infty} b_{n} z^{-n}$ in $\Sigma$. Each configuration $\gamma_{0}$ yields a $\Phi_{0}$ and coeffcients $\pi_{j}$. Of course, equality occurs for $g=\varphi_{0}$.

If we define the functional

$$
\Lambda(g)=\frac{1}{2 \pi i} \int_{|w|=R} g\left(\Phi_{0}(w)\right) w^{k} d w
$$

for any $g$ analytic in $\tilde{\mathbf{D}}$ (and $R$ sufficiently large), then inequality (4.1) says that $\operatorname{Re} \Lambda(g) \leqq 0$ for all $g$ in $\Sigma$, and equality occurs for $g=\varphi_{0}$. In other words, the presence of a $k^{\text {th }}$ order zero on the omitted set of some support point implies that 
$\varphi_{0}$ is a support point associated with the functional $\Lambda$. Since

$$
\Lambda\left(\varphi_{0}^{-j}\right)=\frac{1}{2 \pi i} \int_{|w|=R} \varphi_{0}\left(\Phi_{0}(w)\right)^{-j} w^{k} d w=\frac{1}{2 \pi i} \int_{|w|=R} w^{k-j} d w,
$$

we have $\Lambda\left(\varphi_{0}^{-k-1}\right)=1$ and $\Lambda\left(\varphi_{0}^{-j}\right)=0$ for all $j \neq k+1$. In this case the associated quadratic differential $\Lambda\left(1 /\left(\varphi_{0}-w\right)\right) d w^{2}$ would reduce simply to $w^{k} d w^{2}$.

We shall work out some examples. It is sometimes convenient to use the formula

$$
\begin{gathered}
\pi_{j}=\Lambda\left(z^{-j}\right)=\frac{1}{2 \pi i} \int_{|w|=R} \Phi_{0}(w)^{-j} w^{k} d w=\frac{1}{2 \pi i} \int_{|w|=R} \frac{j}{k+1} \frac{\Phi_{0}^{\prime}(w)}{\Phi_{0}(w)^{j+1}} w^{k+1} d w \\
=\frac{j}{k+1} \frac{1}{2 \pi i} \int_{|z|=R^{\prime}} \frac{\varphi_{0}(z)^{k+1}}{z^{j+1}} d z
\end{gathered}
$$

for any $R^{\prime}>1$.

If $\gamma_{0}$ is the symmetric $k+2$-star, then $\varphi_{0}(z)=z\left(1+z^{-k-2}\right)^{2 /(k+2)}$ and so $\pi_{-1}=$ $-2 /(k+2)$ and $\pi_{j}=0$ for $j=1, \ldots, k$. In this case the inequality (4.2) becomes $\operatorname{Re} b_{k+1} \leqq 2 /(k+2)$. Since this inequality is known ([2]) to be false for all $k \geqq 2$, it follows that the omitted arc $\Gamma$ for any support point cannot have a zero of order $k \geqq 2$ at which it forks in all $k+2$ directions. This is the argument given also in [2].

Next suppose that $\gamma_{0}$ is the real interval $[0,4]$ and $\varphi_{0}(z)=z+2+1 / z$. Then $\pi_{j}=\frac{j}{k+1}\left(\begin{array}{c}2 k+2 \\ k-j+1\end{array}\right)$ for $j=-1,1, \ldots, k$. In order to prove Conjecture 4.1 it is sufficient to disprove inequalities (4.2) in this case. Thus the following conjecture also implies Conjecture 4.1.

Conjecture 4.3. For each $k \geqq 2$, there is a function $g(z)=z+\sum_{n=0}^{\infty} b_{n} z^{-n}$ in $\Sigma$ for which

$$
\operatorname{Re}\left\{b_{k+1}+\sum_{j=1}^{k} \frac{j}{k+1}\left(\begin{array}{c}
2 k+2 \\
k-j+1
\end{array}\right) b_{j}\right\}>\frac{1}{k+1}\left(\begin{array}{c}
2 k+2 \\
k
\end{array}\right) .
$$

For example, for $k=2$ it would be useful to prove that there is a function in $\Sigma$ for which $\operatorname{Re}\left\{b_{3}+4 b_{2}+5 b_{1}\right\}>5$. Even this question remains open.

Finally, suppose that the order $k=2 x$ of the zero is even, that $\gamma_{0}$ is the real interval $[-2,2]$, and that $\varphi_{0}(z)=z+1 / z$. This situation arises when the omitted set for some support point passes straight through a critical point. In this case $\pi_{j}=0$ if $j$ is even and $\pi_{j}=\frac{j}{2 x+1}\left(\begin{array}{c}2 x+1 \\ 1 / 2(2 x-j+1)\end{array}\right)$ if $j$ is odd. Consequently, (4.2) becomes

$$
\operatorname{Re}\left\{b_{2 \varkappa+1}+\sum_{v=1}^{x} \frac{2 v-1}{2 \varkappa+1}\left(\begin{array}{c}
2 \varkappa+1 \\
x-v+1
\end{array}\right) b_{2 v-1}\right\} \leqq \frac{1}{2 \varkappa+1}\left(\begin{array}{c}
2 \varkappa+1 \\
x
\end{array}\right) .
$$

The simplest of these, for $x=1$, corresponding to a zero of order 2 is

$$
\operatorname{Re}\left\{b_{3}+b_{1}\right\} \leqq 1 \text {. }
$$


However, in Corollary 3.2 we proved that this inequality is false. Therefore we have the fcllowing theorem.

Theorem 4.4. Let $f$ be a support point of a linear functional $L$. If the omitted set $\Gamma$ of $f$ contains a second order zero of the quadratic differential $L(1 /(f-w)) d w^{2}$, then no subarc of $\Gamma$ can pass straight through this critical point.

Corollary 4.5. If a support point $f$ of a linear functional $L$ is odd, then its quadratic differential $L(1 /(f-w)) d w^{2}$ cannot reduce to $w^{2} d w^{2}$.

While Theorem 4.4 prevents the omitted arcs of a support point from passing straight through a second order zero, it could stop there or possibly make a left or right turn.

\title{
References
}

[1] AhLfors, L. V.: Conformal invariants. - McGraw-Hill Book Company, New York, 1973.

[2] Chang, A., M. M. Schiffer, and G. Schober: On the second variation for univalent functions. - J. Analyse Math. 40, 1981, 203-238.

[3] ChARZYŃski, Z., and M. SCHIFFer: A geometric proof of the Bieberbach conjecture for the fourth coefficient. - Scripta Math. 25, 1960, 173-181.

[4] Jenkins, J. A.: On certain coefficients of univalent functions. - Analytic functions. Princeton Univ. Press, Princeton, N. J., 1960, 159-194.

[5] Jenkins, J. A.: On normalization in the general coefficient theorem. - Proceedings of the International Congress of Mathematicians 1962. Institut Mittag-Leffler, Ojmsholm, Sweden, 1963, 347-350.

[6] Kirwan, W. E., and G. Schober: New inequalities from old ones. - Math. Z. 180, 1982, 19-40.

[7] KubotA, Y.: A remark on the third coefficient of meromorphic univalent functions. - Kōdai Math. Sem. Rep. 29, 1977, 197-206.

[8] Pfluger, A.: The Fekete-Szegö inequality by a variational method. - Ann. Acad. Sci. Fenn. Ser. A I Math. 10, 1985, 447-454.

[9] SCHAefFer, A. C., and D. C. SPENCER: Coefficient regions for schlicht functions. - American Mathematical Society Colloquium Publications 35. American Mathematical Society, New York, N. Y., 1950.

[10] Schober, G.: Univalent functions-selected topics. - Lecture Notes in Mathematics 478. Springer Verlag, Berlin-Heidelberg-New York, 1975.

[11] SCHOBER, G., and J. K. Williams: On coefficient estimates and conjectures for the class $\Sigma$. Math. Z. 186, 1984, 309-320.

[12] TeIchmüller, O.: Ungleichungen zwischen den Koeffizienten schlichter Funktionen. S.-B. Preuss. Akad. Wiss. Phys.-Math. K1., 1938, 363-375.

\author{
Indiana University \\ Department of Mathematics \\ Bloomington, Indiana 47405 \\ USA
}

Received 5 April 1984 